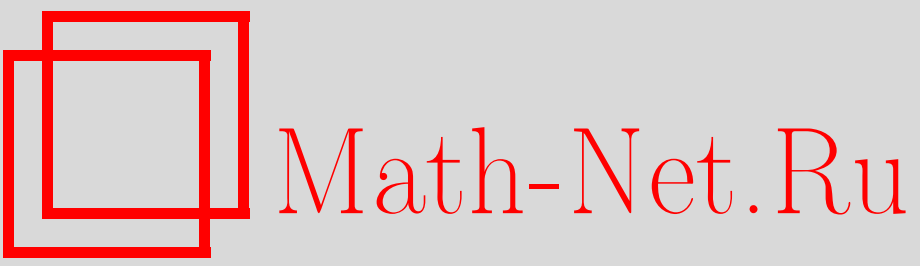

А. В. Павлов, Теорема типа больших уклонений для критерия хи-квадрат, $У M H$, 1996, том 51, выпуск 1, 159-160

DOI: https://doi.org/10.4213/rm932

Использование Общероссийского математического портала Math-Net.Ru подразумевает, что вы прочитали и согласны с пользовательским соглашением

http://www.mathnet.ru/rus/agreement

Параметры загрузки:

IP : 52.90 .164 .192

26 апреля 2023 г., 18:31:38 


\title{
ТЕОРЕМА ТИПА БОЛЬШИХ УКЛОНЕНИЙ ДЛЯ КРИТЕРИЯ ХИ-КВАДРАТ
}

\author{
А.В. ПАвлов
}

В заметке найдена асимптотика ошибки второго рода для критерия хи-квадрат в случае фиксированных простой гипотезы и простой альтернативы при асимптотически постоянной ошибке первого рода. Асимптотика приведена в форме $\exp [-n \Lambda(1+o(1))]$, где $n$ - число опытов. Точньй вид константы $\Lambda$ следует из теоремы 1 . Значение константы $\Lambda$ может быть легко вычислено. Вид константы $\Lambda$ может быть использован для выбора асимптотически оптимального разбиения оси $(-\infty, \infty)$ на непересекающиеся множества, задающие статистику хи-квадрат, причем из теоремы 1 следует, что критерий хи-квадрат является весьма асимптотически эффективным, так как константа $\Lambda$ принимает большое значение для широкого класса разбиений. С помощью подхода, приведенного в данной статье, возможно получение аналогичных результатов для других критериев.

Методы доказательства теоремы 1 существенно опираются на применение условного биномиального распределения к теореме о больших уклонениях для асимптотически нормальных случайных сумм, приведенной в [1].

Определим статистику хи-квадрат. Рассмотрим разбиение оси $(-\infty, \infty)$ на непересекающиеся множества $\left\{\Delta_{i}, i=1, \ldots, k\right\}$. Пусть $d \mathrm{P}_{0}(t)$ - произвольная мера, такая что $\int_{\Delta_{i}} d \mathrm{P}_{0}(t)=$ $p_{0}\left(\Delta_{i}\right) \neq 0, i=1, \ldots, k$. Пусть

$$
\chi^{2}=\sum_{i=1}^{k}\left[\nu\left(\Delta_{i}\right)-n p_{0}\left(\Delta_{i}\right)\right]^{2} / n p_{0}\left(\Delta_{i}\right)
$$

где $\nu\left(\Delta_{i}\right)$ - число элементов выборки $\left\{x_{i}=\xi_{i}(\omega), i=1, \ldots, n\right\}$, принадлежащих $\Delta_{i}, i=$ $1, \ldots, k$. Случайные величины $\left\{\xi_{i}\right\}$ независимы и одинаково распределены. Мы предполагаем, что гипотеза о совпадении набора $\left\{p_{0}\left(\Delta_{i}\right), i=1, \ldots, k\right\}$ с набором вероятностей $\left\{p_{1}\left(\Delta_{i}\right)=\right.$ $\left.\mathrm{P}\left\{\xi_{1} \in \Delta_{i}\right\}, i=1, \ldots, k\right\}$ не верна и оцениваем вероятность ошибки второго рода $\mathrm{P}\left\{\chi^{2} \in[0, \gamma]\right\}$ для фиксированной критической области $(\gamma, \infty)$, предполагаем, что истинный набор вероятностей для выборки $\left\{x_{i}=\xi_{i}\right\}$ известен и $p_{1}\left(\Delta_{i}\right) \neq 0, i=1, \ldots, k$. Обозначим

$$
\begin{gathered}
p(i)=p_{1}\left(\Delta_{i}\right) /\left(1-\sum_{j=1}^{i-1} p_{1}\left(\Delta_{j}\right)\right), \\
\Delta p(i)=p_{0}\left(\Delta_{i}\right)-p_{1}\left(\Delta_{i}\right)\left[\left(1-\sum_{j=1}^{i-1} p_{0}\left(\Delta_{j}\right)\right) /\left(1-\sum_{j=1}^{i-1} p_{1}\left(\Delta_{j}\right)\right)\right] ; \\
H(r+\alpha)=(r+\alpha) \ln ((r+\alpha) / r)-(q-\alpha) \ln ((q-\alpha) / q) \geqslant 2 \alpha^{2}, \quad q+r=1,
\end{gathered}
$$

где $H(r+\alpha)$ - функция больших уклонений [1, с. 131], [2, с. 539]. В теореме 1 роль $r$ будут играть $p_{1}\left(\Delta_{1}\right)$ и $p(i)$.

Теорема 1. Если набор вероятностей $\left\{p_{1}\left(\Delta_{i}\right)=\mathrm{P}\left\{\xi_{1} \in \Delta_{i}\right\}, i=1, \ldots, k\right\}$ для въборки из $n$ независимых одинаково распределенных случайных величин $\left\{\xi_{i}\right\}$ не совпадает с набором вероятностей $\left\{p_{0}\left(\Delta_{i}\right), i=1, \ldots, k\right\}, k>2$, то

$$
\mathrm{P}\left\{\chi^{2} \in[0, \gamma]\right\}=\exp [-\Lambda n(1+o(1))]
$$

əде

$$
\Lambda=H\left(p_{1}\left(\Delta_{1}\right)+\left[p_{0}\left(\Delta_{1}\right)-p_{1}\left(\Delta_{1}\right)\right]\right)+\sum_{i=2}^{k-1}\left(1-\sum_{j=1}^{i-1} p_{0}\left(\Delta_{j}\right)\right) H\left(p(i)+\Delta p_{i}\right)
$$


ДокАЗАТЕЛЬСтво. Введем два вектора $\bar{\varkappa}=\left(\varkappa_{1}, \ldots, \varkappa_{k}\right)$ и $\bar{\beta}=\left(\beta_{1}, \ldots, \beta_{k}\right)$

$$
\begin{gathered}
\varkappa_{i}=\left(\nu\left(\Delta_{i}\right)-n p_{0}\left(\Delta_{i}\right)\right)^{2} / n p_{0}\left(\Delta_{i}\right), \\
G_{i}\left(x_{i}\right)=\mathrm{P}\left\{\varkappa_{i} \leqslant x_{i}\right\}, \quad i=1, \ldots, k ; \\
\beta_{i}=I_{i}(1)+\cdots+I_{i}(\nu(i)), \quad \nu(i)=n-\nu\left(\Delta_{1}\right)-\ldots-\nu\left(\Delta_{i}\right), \\
i=2, \ldots, k-2, \quad \beta_{1}=\nu\left(\Delta_{1}\right),
\end{gathered}
$$

где при фиксированном $i$ последовательность $\left\{I_{i}(j), j=1,2, \ldots\right\}$ состоит из независимых одинаково распределенных случайных величин таких, что $I_{i}(1)=1$ с вероятностью $p(i)=$ $p_{1}\left(\Delta_{i}\right) /\left(1-\sum_{j=1}^{i-1} p_{1}\left(\Delta_{j}\right)\right)$ и $I_{i}(1)=0$ с вероятностью $1-p(i), i=2, \ldots, k-2$. Пусть $A-$ событие:

$$
A=\left\{n p_{0}\left(\Delta_{i}\right)-c n^{1 / 2}<\nu(i)<n p_{0}\left(\Delta_{i}\right)+c n^{1 / 2}, i=1, \ldots, k-1\right\} .
$$

Очевидно, при некоторой константе $c \quad A \in\left\{\chi^{2} \in[0, \gamma]\right\}$.

Выполнено тождество

$$
\begin{aligned}
\mathrm{P}\left\{\varkappa_{i} \leqslant x_{i} \mid \varkappa_{1}=x_{1} \cap \cdots \cap \varkappa_{i-1}=x_{i-1} \cap A\right\} & \\
= & \mathrm{P}\left\{\left[\beta_{i}-p(i) \nu_{x}(i) \leqslant\left(n p_{0}\left(\Delta_{i}\right)\right)^{1 / 2} x_{i}\right.\right. \\
& \left.\left.+\sum_{j=1}^{i-1} x_{j} p(i)\left(n p_{0}\left(\Delta_{j}\right)\right)^{1 / 2}+n p_{0}\left(\Delta_{i}\right)-n p(i)\left(1-\sum_{j=1}^{i-1} p_{0}\left(\Delta_{j}\right)\right)\right] \cap A\right\}, \\
i & =2, \ldots, k-2,
\end{aligned}
$$

где

$$
\nu_{x}(i)=\nu(i)=n \sum_{j=i}^{n} p_{0}\left(\Delta_{j}\right)-\sum_{j=1}^{i-1} x_{j}\left(n p_{0}\left(\Delta_{j}\right)\right)^{1 / 2} .
$$

Обозначим коэффициенты при $n^{1 / 2}$ в правой части неравенства через $c_{i}\left(x_{1}, \ldots, x_{i}\right)$, а разность $p_{0}\left(\Delta_{i}\right)-p(i)\left(1-\sum_{j=1}^{i-1} p_{0}\left(\Delta_{j}\right)\right)$ - через $\Delta p_{i}$. Несложно проверить, что [3], [4]

$$
\begin{aligned}
& \mathrm{P}\left\{\chi^{2} \in[0, \gamma]\right\}=\int_{J \cap 0 \leqslant x_{1}^{2} \leqslant \gamma} d G_{1}\left(c_{1}\left(x_{1}\right) n^{1 / 2}+n\left[p_{0}\left(\Delta_{1}\right)-p_{1}\left(\Delta_{1}\right)\right]\right) \\
& \quad \times \int_{0 \leqslant x_{2}^{2}<\gamma-x_{1}^{2} \cap J} d F_{2}\left(c_{2}\left(x_{1}, x_{2}\right) n^{1 / 2}+n \Delta p_{2}\right) \\
& \quad \times \int \ldots \int_{0 \leqslant x_{k-1}^{2} \leqslant \gamma-x_{1}^{2}-\ldots-x_{k-2}^{2} \cap J} d F_{k-1}\left(c_{k-1}\left(x_{1}, \ldots, x_{k-1}\right) n^{1 / 2}+n \Delta p_{k-1}\right),
\end{aligned}
$$

где

$$
\begin{gathered}
J:\left\{\left(A_{1} x_{1}+\cdots+A_{k-1} x_{k-1}\right)^{2}<\gamma-x_{1}^{2}-\ldots-x_{k-1}^{2}\right\}, \\
A_{j}=\left(p_{1}\left(\Delta_{j}\right) / p_{0}\left(\Delta_{j}\right)\right)^{1 / 2}, \quad j=1, \ldots, k-1, \\
F_{j}(t)=\mathrm{P}\left\{\beta_{j}-p(i) \nu_{x}(i) \leqslant t\right\} .
\end{gathered}
$$

Применяя теорему о больших уклонениях [1, с. 205], получаем, что все члены с $n^{1 / 2}$ для нашей асимптотики не существенны и теорема 1 доказана.

\section{СПИСОК ЛИТЕРАТУРЫ}

[1] Боровков А. А. Теория вероятностей. М.: Наука, 1986. [2] Ширяев А. Н. Вероятность. М.: Наука, 1989. [3] Павлов А. В. // Теория вероятн. и ее примен. 1990. Т. 35. С. 716-726. [4] Павлов А.В. Задачи по математической статистике. М.: Изд-во МИРЭА, 1993. ISBN 5-230-12148-3. 